\title{
Factorization method for some inhomogeneous Liénard equations
}

\author{
O. Cornejo-Pérez \\ Facultad de Ingeniería, Universidad Autónoma de Querétaro, \\ Centro Universitario Cerro de las Campanas, 76010 Santiago de Querétaro, México. \\ e-mail: octavio.cornejo@uaq.mx \\ S. C. Mancas \\ Department of Mathematics, Embry-Riddle Aeronautical University, \\ Daytona Beach, FL 32114-3900, USA \\ e-mail:mancass@erau.edu \\ H. C. Rosu \\ IPICyT, Instituto Potosino de Investigación Científica y Tecnológica, \\ Camino a la presa San José 2055, Col. Lomas 4 a Sección, 78216 San Luis Potosí, S.L.P., México. \\ e-mail: hcr@ipicyt.edu.mx \\ C. A. Rico-Olvera \\ Facultad de Ingeniería, Universidad Autónoma de Querétaro, \\ Centro Universitario Cerro de las Campanas, 76010 Santiago de Querétaro, México. \\ e-mail: crico24@alumnos.uaq.mx
}

Received 4 January 2021; accepted 18 January 2021

\begin{abstract}
We obtain closed-form solutions of several inhomogeneous Liénard equations by the factorization method. The two factorization conditions involved in the method are turned into a system of first-order differential equations containing the forcing term. In this way, one can find the forcing terms that lead to integrable cases. Because of the reduction of order feature of factorization, the solutions are simultaneously solutions of first-order differential equations with polynomial nonlinearities. The illustrative examples of Liénard solutions obtained in this way generically have rational parts, and consequently display singularities.
\end{abstract}

Keywords: Factorization; inhomogeneous; Liénard equation; Abel equation; Riccati equation.

PACS: 02.30.Hq; 02.30.Ik

DOI: https://doi.org/10.31349/RevMexFis.67.443

\section{Introduction}

The exact solutions of nonlinear ordinary differential equations (ODEs) describe the behavior of a great variety of physical, chemical, biological, and engineering systems. Widespread systems in these vast areas of research can be described by homogeneous Liénard equations, which have been intensively studied over the years, see, e.g., [1] and the recent review [2]. On the other hand, the same type of inhomogeneous equations received relatively less attention despite the remarkable leap forward brought by the discovery of an irregular noise, later termed deterministic chaos, in the case of sinusoidally driven triode circuits by van der Pol and van der Mark in 1927 [3]. Our focus in this short paper is on inhomogeneous Liénard type equations of the form

$$
\ddot{u}+G(u) \dot{u}+F(u)=I(t),
$$

where the dot denotes the time derivative, $d / d t, G(u)$ and $F(u)$ are arbitrary, but usually polynomial, functions of $u$, and the forcing term $I(t)$ is an arbitrary continuous function of time.

The main goal of the present paper is to show how the factorization method developed in [4-6] and the factorization conditions thereof can be used to obtain some integrable inhomogeneous Liénard equations for specific forcing terms. The key point is that the factorization method helps to reduce the inhomogeneous Liénard equations to first-order nonlinear equations, such as Abel and Riccati equations, which are presumably easier to solve in some cases. We recall that the reduction to Riccati equations of the linear Schrödinger equations has been extensively used in supersymmetric quantum mechanics and older factorization methods as reviewed in $[7,8]$.

\section{The nonlinear factorization}

As in [4-6], we consider the factorization of (1)

$$
\left[\frac{d}{d t}-f_{2}(u)\right]\left[\frac{d}{d t}-f_{1}(u)\right] u=I(t)
$$


under the conditions

$$
\begin{aligned}
f_{2}+\frac{d\left(f_{1} u\right)}{d u} & =-G(u) \\
f_{1} f_{2} u & =F(u),
\end{aligned}
$$

adding the scheme proposed in [9], where one assumes $\left[d / d t-f_{1}(u)\right] u=\Omega(t)$. This yields the following coupled ODEs for (2),

$$
\begin{aligned}
\dot{\Omega}-f_{2}(u) \Omega & =I(t) \\
\dot{u}-f_{1}(u) u & =\Omega(t),
\end{aligned}
$$

which we further simplify by taking the second factorizing function as a constant, $f_{2}=a_{2} \equiv$ const.,

$$
\begin{aligned}
\dot{\Omega}-a_{2} \Omega & =I(t) \\
\dot{u}-f_{1}(u) u & =\Omega(t) .
\end{aligned}
$$

Besides, using the constant function $f_{2}$, conditions (3) and (4) imply a relationship between functions $F$ and $G$ given by

$$
F(u)=-a_{2}\left(c_{2}+a_{2} u+\int^{u} G(u) d u\right),
$$

where $c_{2}$ stands for an integration constant, or equivalently

$$
G(u)=-\left(\frac{1}{a_{2}} \frac{d F}{d u}+a_{2}\right) .
$$

Denoting $\mathcal{I}(t)=\int_{0}^{t} e^{-a_{2} t} I(t) d t$, the solution to (7) is

$$
\Omega(t)=e^{a_{2} t}\left[c_{1}+\mathcal{I}(t)\right],
$$

where $c_{1}$ is an integration constant given by $c_{1}=\Omega(0)$. This allows to rewrite $(\underline{8)}$ in the form

$$
\dot{u}=\frac{1}{a_{2}} F(u)+e^{a_{2} t}\left[c_{1}+\mathcal{I}(t)\right],
$$

whose general solution is also the solution of the Liénard Eq. (1), while further particular solutions can be obtained by setting $c_{1}=0$.

Viceversa, one can say that (12) is a first-order nonlinear reduction of forced Liénard equations of the form

$$
\ddot{u}-\left(\frac{1}{a_{2}} \frac{d F}{d u}+a_{2}\right) \dot{u}+F(u)=I(t) .
$$

Thus, integrable cases of (12) can provide Liénard solutions in closed form. Since among the most encountered forced Liénard equations are these having $F(u)$ in the form of cubic and quadratic polynomials, in the rest of the paper, we address the applications of this solution method to some cases of these types.

\section{The inhomogeneous Duffing-van der Pol os- cillator}

We choose the particular cubic case $F(u)=A u+C u^{3}$ because it corresponds to the forced Duffing-van der Pol oscillator [10]

$$
\ddot{u}-\left[\left(a_{2}+A / a_{2}\right)+3\left(C / a_{2}\right) u^{2}\right] \dot{u}+A u+C u^{3}=I(t) .
$$

This equation admits the factorization

$$
\left[\frac{d}{d t}-a_{2}\right]\left[\frac{d}{d t}-\left(\alpha+\gamma u^{2}\right)\right] u=I(t),
$$

where $\alpha=A / a_{2}$ and $\gamma=C / a_{2}$.

The corresponding first-order equation is the Abel equation

$$
\dot{u}=\gamma u^{3}+\alpha u+\Omega(t)
$$

The change of variables

$$
u=y e^{\alpha t}, \quad x=\frac{\gamma}{2 \alpha} e^{2 \alpha t},
$$

turns $(16)$ into the normal form

$$
\frac{d y}{d x}=y^{3}+\mathcal{N}(x)
$$

with invariant

$$
\mathcal{N}(x)=\frac{1}{\gamma} e^{\left(a_{2}-3 \alpha\right) t(x)}\left[c_{1}+\mathcal{I}(t(x))\right] .
$$

Unfortunately, this formula shows that inhomogeneous Abel equations in this category are not integrable by the separation of variables because $\mathcal{N}(x)$ cannot be made constant as required by this type of integrability. Only in the force-free particular case $I(t)=0$, the invariant can be reduced to the constant

$$
\mathcal{N}_{0}=\frac{c_{1}}{\gamma}
$$

By separation of variables, the solution is given by the implicit relation

$$
\begin{aligned}
\ln & {\left[\frac{\left(\sqrt[3]{\mathcal{N}_{0}}+y\right)^{2}}{\mathcal{N}_{0}^{2 / 3}-\sqrt[3]{\mathcal{N}_{0}} y+y^{2}}\right]-2 \sqrt{3} \tan ^{-1}\left[\frac{1-\frac{2}{\sqrt[3]{\mathcal{N}_{0}}} y}{\sqrt{3}}\right] } \\
& =6 \mathcal{N}_{0}^{2 / 3}\left(x+c_{2}\right)
\end{aligned}
$$

This solution has been obtained previously in [10].

\section{Quadratic inhomogeneous Liénard equa- tions}

If we set $F(u)=A u+B u^{2}$, then the first order equivalent equation is the Riccati equation

$$
\dot{u}=\beta u^{2}+\alpha u+\Omega(t), \quad \beta=B / a_{2} .
$$

Equation (22) can be transformed into the normal form [11]

$$
\dot{z}=z^{2}+\mathcal{N}(t)
$$


where

$$
z(t)=\beta u(t)+\frac{\alpha}{2}, \quad \mathcal{N}(t)=\beta \Omega(t)-\frac{\alpha^{2}}{4} .
$$

For integrable cases of separable type, one should have $\mathcal{N}(t)$ as an arbitrary real constant that we choose $p^{2} / 4$, implying $\Omega(t)=\left(p^{2}+\alpha^{2}\right) / 4 \beta$ also a constant, as well as a constant driving force

$$
I(t)=-\frac{a_{2}}{\beta}\left(\frac{p^{2}+\alpha^{2}}{4}\right) .
$$

In this simple case, we obtain a Liénard solution of (13) of the form

$$
u(t)=-\frac{\alpha}{2 \beta}\left[1-\frac{p}{\alpha} \tan \left(\frac{p}{2}\left(t+c_{2}\right)\right)\right] .
$$

\subsection{Linear polynomial source term}

After the constant driving case, it is orderly to consider the source term as the linear polynomial $I(t)=t+\delta$, where $\delta$ is an arbitrary constant. We set $a_{2}=1$ and $c_{1}=0$, and we obtain the Riccati equation

$$
\dot{u}=\beta u^{2}+\alpha u-(t+\tilde{\delta}), \quad \tilde{\delta}=\delta+1
$$

with solution given by

$$
u(t)=-\frac{\alpha}{2 \beta}\left[1+\frac{\beta^{\frac{1}{3}}}{\alpha} \frac{k_{2} A i^{\prime}(\tilde{t})+B i^{\prime}(\tilde{t})}{k_{2} A i(\tilde{t})+B i(\tilde{t})}\right],
$$

where $\tilde{t}=\beta^{1 / 3}\left[\alpha^{2} / 4 \beta+(t+\tilde{\delta}]\right.$, the prime denotes the $\tilde{t}$ derivative, and $k_{2}$ is an integration constant. However, the presence of the rational term in Airy functions turns singular such as Liénard solutions.

\subsection{Quadratic polynomial source term}

Let the source term be the quadratic polynomial of type $I(t)=a_{2} \beta t^{2}+\left(a_{2} \alpha-2 \beta\right) t-\left(a_{2}+\alpha\right)$. According to Eqs. (11) and (22), and by setting $c_{1}=0$, we have the Riccati equation

$$
\dot{u}=\beta u^{2}+\alpha u-\beta t^{2}-\alpha t+1 .
$$

This equation has the particular solution $u(t)=t$, while the general solution is given by

$$
u(t)=t-\frac{e^{t(\alpha+\beta t)}}{k_{1} \beta+e^{t(\alpha+\beta t)} \sqrt{\beta} \mathcal{F}\left(\frac{\alpha+2 \beta t}{2 \sqrt{\beta}}\right)},
$$

where $\mathcal{F}(x)=e^{-x^{2}} \int_{0}^{x} e^{y^{2}} d y$ is the Dawson integral, and $k_{1}$ is an integration constant. Again, because of the rational term, this solution is singular at $-e^{t(\alpha+\beta t)} \beta^{-1 / 2} \mathcal{F}(\alpha+2 \beta t / 2 \sqrt{\beta})=k_{1}$.

\subsection{Exponential source term}

For the source term of exponential form, $I(t)=\kappa e^{\lambda t}$, and for $c_{1}=0$, the Riccati equation is

$$
\dot{u}=\beta u^{2}+\alpha u+\frac{\kappa}{\lambda-a_{2}} e^{\lambda t} .
$$

The solution is given by

$$
\begin{aligned}
u(t) & =\frac{\alpha}{2 \beta}\left[\frac{\lambda}{\alpha}\right. \\
& \left.\times \frac{2 k_{4} \Gamma\left(1-\frac{\alpha}{\lambda}\right) \tilde{t} J_{1-\frac{\alpha}{\lambda}}(2 \tilde{t})-\tilde{t}^{\frac{\alpha}{\lambda}} \bar{F}(\tilde{t})}{k_{4} \Gamma\left(1-\frac{\alpha}{\lambda}\right) J_{-\frac{\alpha}{\lambda}}(2 \tilde{t})+\Gamma\left(1+\frac{\alpha}{\lambda}\right) J_{\frac{\alpha}{\lambda}}(2 \tilde{t})}\right],
\end{aligned}
$$

where $k_{4}$ is an integration constant, $\tilde{t}=$ $2\left(\sqrt{\kappa \beta} / \lambda \sqrt{\lambda-a_{2}}\right) e^{\lambda t / 2}$, and $\bar{F}(\tilde{t})$ is the following combination of hypergeometric functions

$$
\begin{aligned}
\bar{F}(\tilde{t}) & =\tilde{t}^{2}{ }_{0} F_{1}\left(; 2+\frac{\alpha}{\lambda} ;-\tilde{t}^{2}\right) \\
& +\frac{\alpha}{\lambda}{ }_{0} F_{1}\left(; 1+\frac{\alpha}{\lambda} ;-\tilde{t}^{2}\right)+{ }_{0} F_{1}\left(; \frac{\alpha}{\lambda} ;-\tilde{t}^{2}\right) .
\end{aligned}
$$

For $k_{4}=0$, we have the simpler solution

$$
\begin{aligned}
& u(t)=-\frac{\alpha}{2 \beta} \\
& \times\left[1+\frac{\lambda}{\alpha} \frac{\tilde{t}^{2}{ }_{0} F_{1}\left(; 2+\frac{\alpha}{\lambda} ;-\tilde{t}^{2}\right)+{ }_{0} F_{1}\left(; \frac{\alpha}{\lambda} ;-\tilde{t}^{2}\right)}{{ }_{0} F_{1}\left(; 1+\frac{\alpha}{\lambda} ;-\tilde{t}^{2}\right)}\right] .
\end{aligned}
$$

The case corresponding to $\alpha=-1$ simplifies further to

$$
u(t)=\frac{e^{t}}{\sqrt{\beta}} \tan \left[\sqrt{\beta}\left(e^{t}+k_{4}\right)\right] .
$$

\subsection{Back to the constant source case}

We return to the constant source term case since we wish to point out the interesting feature that it is more general than the exponential case. Indeed, let us take the source term as $I(t)=\epsilon$, an arbitrary constant, and $a_{2}=1$. This leads to the Riccati equation

$$
\dot{u}=\beta u^{2}+\alpha u+c_{1} e^{t}-\epsilon,
$$

which is similar to the Riccati equation for the exponential case unless for $\epsilon$. The general solution of (35) is a rational expression in terms of Bessel functions given by

$$
\begin{aligned}
u(t) & =\frac{\alpha}{2 \beta}\left[\frac{m}{\alpha} \frac{k_{3}(\alpha-m) \Gamma(-m) J_{-m}(\tilde{t})-(\alpha+m) \Gamma(m) J_{m}(\tilde{t})}{k_{3} \Gamma(1-m) J_{-m}(\tilde{t})+\Gamma(1+m) J_{m}(\tilde{t})}\right] \\
& +\frac{\alpha}{2 \beta}\left[\frac{\tilde{t}}{\alpha} \frac{\left.k_{3} \Gamma(1-m) J_{1-m}(\tilde{t})+m \Gamma(m) J_{1+m}(\tilde{t})\right)}{k_{3} \Gamma(1-m) J_{-m}(\tilde{t})+\Gamma(1+m) J_{m}(\tilde{t})}\right]
\end{aligned}
$$

where $m=\sqrt{\alpha^{2}+4 \beta \epsilon}, \tilde{t}=2 \sqrt{\beta c_{1}} e^{t / 2}$, and $k_{3}$, an integration constant. It displays singularities at the zeros of its denominators.

When $k_{3}=0$, this solution takes the simpler form

$$
u(t)=-\frac{\alpha}{2 \beta}\left[\left(1+\frac{m}{\alpha}\right)-\frac{\tilde{t}}{\alpha} \frac{J_{m+1}(\tilde{t})}{J_{m}(\tilde{t})}\right] .
$$


Notice that in the particular case of $c_{1}=0$, the exponential scaling of time is annihilated, and the Riccati equation is of constant coefficients having the well-known regular kink solution

$$
u(t)=-\frac{\alpha}{2 \beta}\left[1+\frac{m}{\alpha} \tanh \left(\frac{m}{2}\left(t+k_{3}\right)\right)\right],
$$

which is also a Liénard kink. If in the expression for the parameter $m$, we substitute $\epsilon$ by (25) for $a_{2}=1$, we obtain $m=i p$, and (38) becomes the solution (26).

\section{Conclusion}

The nonlinear factorization method developed in [4-6,9] has been used to obtain closed-form solutions of certain types of inhomogeneous Liénard equations. The conditions imposed upon the nonlinear coefficients of the equations by the factorization method and the insertion of the forcing term in the factorization scheme act as designing tools of specific forms of the forcing terms to generate integrable cases by these means. The illustrative examples have been chosen from the class of polynomial (up to cubic) and exponential forcing terms similar to a recent study of inhomogeneous Airy equations [12]. However, the obtained Liénard solutions have rational parts, which make them prone to the presence of singularities. The only regular solutions we have obtained by employing this simple factorization method are the usual tanh kinks. Finally, the scheme presented here is bounded to constant factorization functions $f_{2}$, since only in this case equation (5) can be turned into the linear Eq. (7) in the independent variable $t$.
1. M. Lakshmanan and S. Rajasekar, Nonlinear Dynamics: Integrability, Chaos, and Patterns, Springer, Heidelberg 2003. https://doi.org/10.1007/978-3-642-55688-3

2. T. Harko and S.-D. Liang, Exact solutions of the Liénard and generalized Liénard type ordinary nonlinear differential equations obtained by deforming the phase space coordinates of the linear harmonic oscillator, J. Eng. Math. 98 (2016) 93. https://doi.org/10.1007/s10665-015-9812-z

3. B. van der Pol and J. van der Mark, Frequency demultiplication, Nature 120 (1927) 363. https://doi.org/10. $1038 / 120363 \mathrm{a} 0$

4. H.C. Rosu and O. Cornejo-Pérez, Supersymmetric pairing of kinks for polynomial nonlinearities, Phys. Rev. E 71 (2005) 046607. https://doi.org/10.1103/PhysRevE.71. 046607

5. O. Cornejo-Pérez and H.C. Rosu, Nonlinear second order ode's: Factorizations and particular solutions, Prog. Theor. Phys. 114 (2006) 533. https://doi.org//10.1143/ PTP.114.533

6. H.C. Rosu, O. Cornejo-Pérez, M. Pérez-Maldonado, J.A. Belinchón, Extension of a factorization method of nonlinear second order ODE's with variable coefficients, Rev. Mex. Fís. 63 (2017) 218. ISSN: 0035-001X
7. S.-H. Dong, Factorization Method in Quantum Mechanics, Springer, Dordrecht 2007. https://doi.org/10.1007/ 978-1-4020-5796-0]

8. B. Mielnik and O. Rosas-Ortiz, Factorization: little or great algorithm?, J. Phys. A: Math. Gen. 37 (2004) 10007. https: //doi.org/10.1088/0305-4470/37/43/001

9. D.S. Wang and H. Li, Single and multi-solitary wave solutions to a class of nonlinear evolution equations, J. Math. Anal. Appl. 343 (2008) 273. https : / / doi .org/10.1016/j.jmaa . 2008.01 .039

10. V.K. Chandrasekar, M. Senthilvelan, and M. Lakshmanan, New aspects of integrability of force-free Duffing-van der Pol oscillator and related nonlinear systems, J. Phys. A: Math. Gen. 37 (2004) 4527. https://doi.org/10. 1088/0305-4470/37/16/004

11. H. Sarafian, A closed form solution to a special normal form of Riccati equation, Advances in Pure Mathematics 1 (2011) 295. https://doi.org/10.4236/apm.2011.15053

12. T. M. Dunster, Asymptotic solutions of inhomogeneous differential equations having a turning point, Stud. Appl. Math. 145 (2020) 500. https://doi.org/10.1111/sapm. 12326 